\title{
Juxtacortical chondromyxoid fibroma in the small bones: two cases with unusual location and a literature review
}

\author{
Sun-Ju Oh${ }^{1}$, So Hak Chung ${ }^{2}$ \\ Departments of ${ }^{1}$ Pathology and ${ }^{2}$ Orthopedic Surgery, Kosin University Gospel Hospital, Kosin University College of Medicine, Busan, Korea
}

\begin{abstract}
Chondromyxoid fibroma is a rare bone tumor of cartilaginous origin, representing less than $1 \%$ of all bone tumors. It preferentially arises in the eccentric location of the metaphysis of a long tubular bone. Juxtacortical locations are reported infrequently in the long bones and even more rarely in short tubular bones, with only three cases documented. Here we present two new cases of juxtacortical chondromyxoid fibroma in the small bones. One was an intracortical osteolytic lesion of the metatarsal bone of the foot with degenerative atypia that histologically should be differentiated from chondrosarcoma. The other was a phalangeal mass protruding into the interphalangeal joint of the hand, which had been labeled mistakenly as a soft tissue mass preoperatively. These cases illustrated that chondromyxoid fibromas have various the manifestations and should be included in the differential diagnosis of an osteolytic lesion or an exophytic mass in the small bones.
\end{abstract}

Key Words: Chondromyxoid fibroma; Small bones; Juxtacortical

Received: October 11, 2021 Revised: December 8, 2021 Accepted: December 8, 2021

Corresponding Author: Sun-Ju Oh, MD, Department of Pathology, Kosin University Gospel Hospital, Kosin University College of Medicine, 262 Gamcheon-ro, Seo-gu, Busan 49267, Korea

Tel: +82-51-990-6333, Fax: +82-51-990-3080, E-mail: 10highpowerfield@gmail.com

Chondromyxoid fibroma (CMF) is a rare, benign neoplasm of the bones, mostly affecting people in the second or third decade of life, with a slight male predominance. Long bones, especially the proximal tibia, are the most frequently affected sites, followed by the distal femur, pelvis, and small bones of acral sites [1,2]. The typical CMF eccentrically arises in the medulla of the metaphysis, with sharply circumscribed margins. Infrequent cases of juxtacortical subtypes have been documented in long bones, but only three of the short tubular bones have been reported in the English literature (Table 1). Here we present two new cases of juxtacortical CMF in the hand and foot, respectively.

\section{CASE REPORT}

\section{Case 1}

A 43-year-old man presented with a swelling on the dorsum of the right foot for more than one year. He complained of intermittent pain for a few months. Plain films demonstrated a wellmarginated osteolytic lesion on the second metatarsal bone of the right foot (Fig. 1A). Magnetic resonance imaging (MRI) showed a $2 \times 1.7 \mathrm{~cm}$ intracortical mass with expansile growth (Fig. 1B). Medullary bone was partially involved. T1-weighted images revealed an intermediate signal, while T2-weighted images showed a heterogeneous signal with some areas of high intensity. Preoperative diagnosis was a benign bone tumor such as simple bone cyst, and curettage with bone graft was performed.

Histological examination of the lesion showed a lobular architecture on the low-power view (Fig. 2A). Lobules demonstrated hypocellular centers with hypercellular peripheries. Center of the lobules consisted of stellate or spindle-shaped cells with eosinophilic cytoplasmic processes in a myxoid background (Fig. 2B). Cellular areas in the periphery contained round cells with enlarged, hyperchromatic, and pleomorphic nuclei (Fig. 2C). Vacuolization was observed. The nucleocytoplasmic ratio of these cells, however, was low, and no mitosis was found, which implied degeneration rather than malignancy. Overall histologic features rendered a diagnosis of chondromyxoid fibroma. There has been no recurrence for seven months after the surgery. 
Table 1. Reported cases of juxtacortical chondromyxoid fibroma in the small bones

\begin{tabular}{lcll}
\hline Study & Age (yr)/Sex & & Location \\
\hline Baker et al. [2] & 65/M & Foot; metacarpal; NA & Clinical presentation \\
Slotcavage et al. [9] & 29/F & Hand; metacarpal, proximal phalanx; epiphysis, metacarpophalangeal joint & Pain, swelling, movement limitation \\
Han et al. [7] & 17/M & Foot; metatarsal; diaphysis & Pain \\
Present case 1 & 43/M & Foot; metatarsal; metadiaphysis & Pain, swelling \\
Present case 2 & 43/M & Hand; middle phalanx; epiphysis, interphalangeal joint & Pain, movement limitation \\
\hline
\end{tabular}

M, male; NA, not available; F, female.
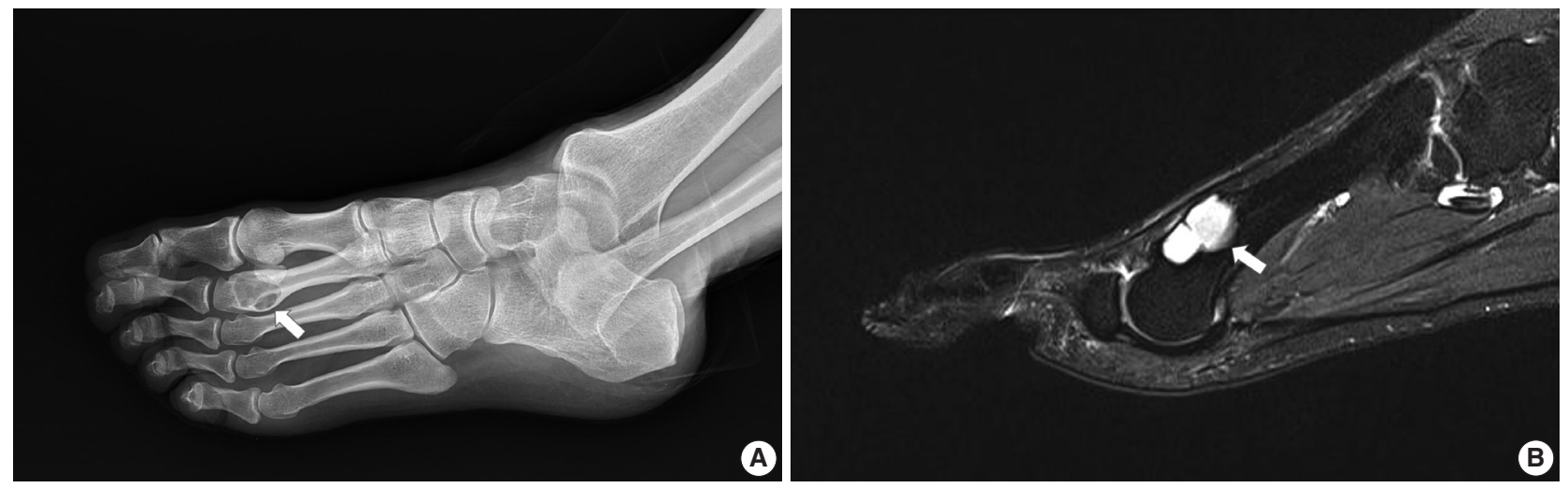

Fig. 1. Radiologic images of the case 1. (A) Oblique radiograph of the foot shows an intracortical lytic lesion (arrow) in the right second metatarsal neck. (B) Sagittal magnetic resonance image shows a hyperintense mass (arrow) with an intact shell of cortical bone.
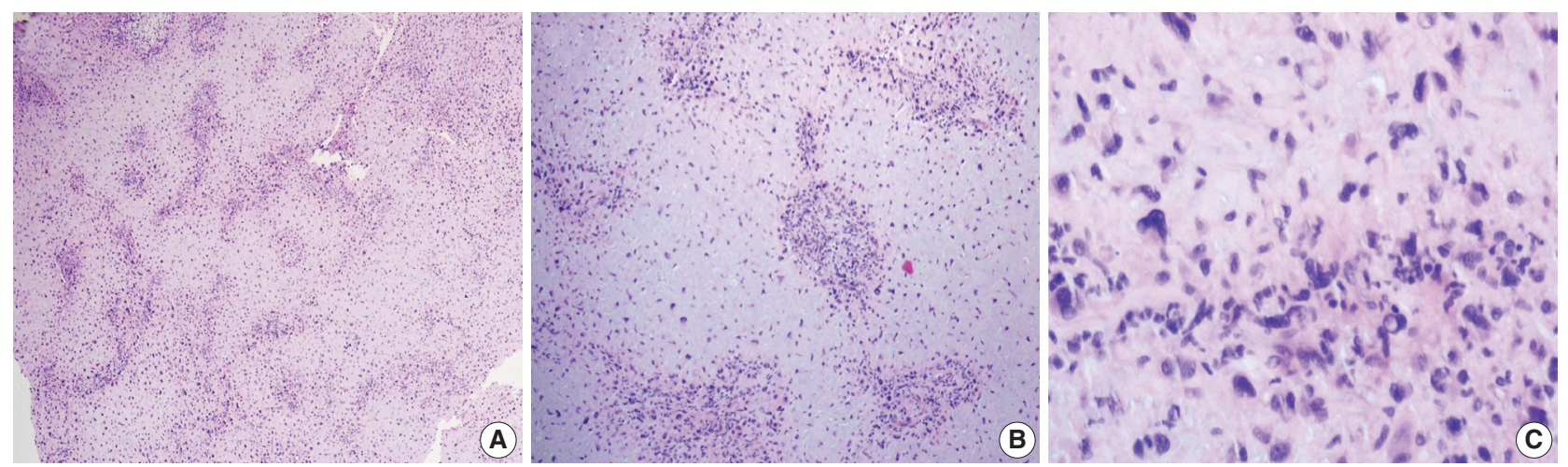

Fig. 2. Histologic findings of the case 1. (A) Low-power view of the tumor shows a prominent lobular patten. (B) The lobules consist of a hypocellular center with abundant myxoid matrix and condensation of tumor cells toward the periphery. (C) The tumor cells in the hypercellular periphery reveal degenerative features including bizarre nuclei, hyperchromasia, and vacuolization.

\section{Case 2}

A 43-year-old man presented with dull pain and limitation of movement on the right index finger without any precipitating trauma or injury. Physical examination revealed a soft tissue swelling in the volar aspect of the proximal interphalangeal joint.

Radiographs of the right hand revealed a soft tissue shadow in the proximal interphalangeal joint of the index finger with a small osteolytic lesion in the middle phalanx (Fig. 3A). Multiple calcific deposits were observed. MRI showed a soft tissue mass with high signal intensity in the proximal interphalangeal joint (Fig. 3B). The lesion eroded the epiphysial cortex of the adjacent mid- dle phalanx and was excised under preoperative diagnosis of synovial chondromatosis. In the surgical field, the mass protruded from the head of the middle phalanx and extended toward the soft tissue. A low-magnification view revealed a lobular pattern of tumor cells with zonal architecture (Fig. 3C). The center of the lobules was hypocellular with round and spindle cells, and the periphery was hypercellular containing round cells and multinucleated giant cells (Fig. 3D). One characteristic feature was numerous spotty calcifications, which corresponded to the preoperative radiographs. The histologic diagnosis was juxtacortical chondromyxoid fibroma. 

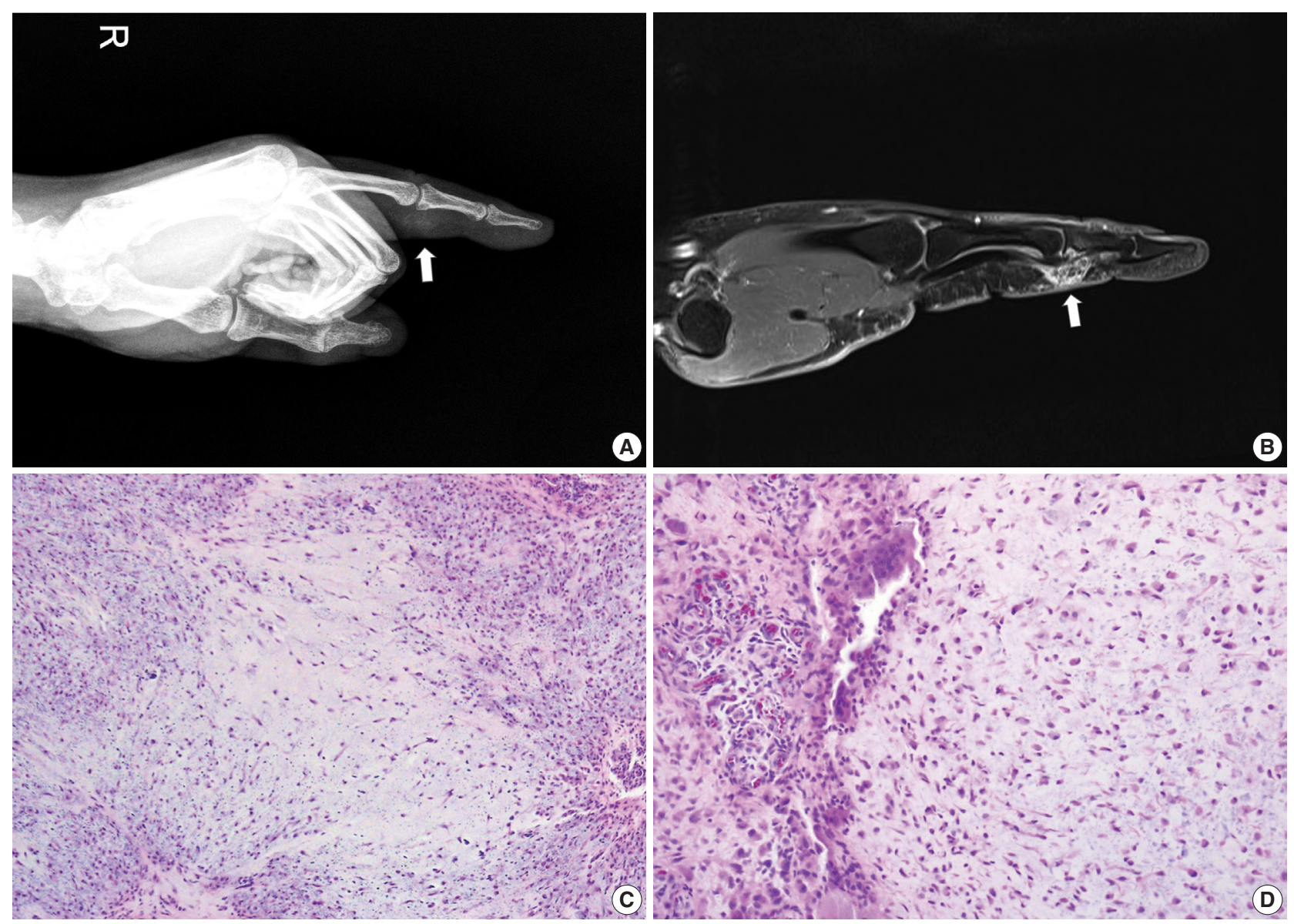

Fig. 3. Radiologic and histologic images of the case 2. (A) Lateral radiograph of the right index finger reveals a soft tissue mass (arrow) with calcification in the proximal interphalangeal joint. The lesion erodes the epiphysis of the mid phalanx. (B) Magnetic resonance image shows a protruding mass (arrow) with heterogeneous intensity. (C) Microphotographs show a characteristic lobular growth pattern. Spotty calcifications are scattered throughout the lesion. (D) Hypercellular area surrounding the hypocellular myxoid lobules occasionally contains multinucleated giant cells.

\section{DISCUSSION}

$\mathrm{CMF}$ is a rare benign bone tumor, first described by Jaffee and Lichtenstein in 1948 [3]. It accounts for less than $1 \%$ of all bone tumors and shows slight male predominance in the second and third decades of life [1]. CMF has a predilection for the metaphysis of a long bone, most often the proximal tibia. Small bones of the feet or flat bones of the ilium are also commonly involved. However, vertebrae, ribs, scapula, skull, and jawbones are rarely involved.

The tumor typically has eccentric location in the bones, with a few cases of juxtacortical subtypes [2,4-9]. Juxtacortical CMFs encompass all surface-type tumors including cortical, subperiosteal, periosteal, or parosteal lesions, and the exact origin is not always identified [2]. Of these juxtacortical cases, most involve long bones, with only three cases affecting small bones of the hands or feet (Table 1) $[2,7,9]$. Four of five cases including ours involved metacarpal or metatarsal bones. Interestingly, two cases involving the hand appeared in the form of an exophytic mass that involved the joint space; the second current case was mistaken for a soft tissue mass invading the bone on the preoperative radiologic examination.

Pain is the most common symptom in CMFs, as is true of the juxtacortical type of small bones. In addition, soft tissue swelling can manifest due to the thin musculature of hands and feet, and range of motion can be limited when joints are involved.

Histologic features of CMF show a characteristic lobular architecture of a hypocellular center with condensation of tumor cells toward the periphery. The center consists of chondroid and myxoid matrix, containing stellate or spindle-shaped cells often with eosinophilic cytoplasmic process. The lobule is surrounded by fibrous periphery with round cells and scattered multinucleated giant cells. CMFs rarely are accompanied by calcification, but 
juxtacortical types can show calcification in the coarse pattern [2]. Tumor cells in CMF are pleomorphic with large nuclei in approximately $20 \%$ of cases [10]. Such pleomorphism can be prominent, as in one of our cases, and should be distinguished from chondrosarcoma. The differential findings of CMF are no alteration of nucleocytoplasmic ratio and absence or rarity of unequivocal mitoses.

The main differential diagnosis of CMF is chondrosarcoma, especially with accompanying pleomorphism. The radiologic findings of chondrosarcoma differ markedly from sharply defined CMF even when it slightly erodes the bone. Histologically, chondrosarcoma reveals hypercellularity without zonal architecture. The CMF also differs from chondrosarcoma, with the latter usually producing a large amount of well-differentiated hyaline cartilage and entrapping the pre-existing lamellar bone trabeculae with permeation [11].

Juxtacortical CMFs, especially on the long bones, should be differentiated from the other ominous cartilage-containing neoplasms such as periosteal osteosarcoma and parosteal osteosarcoma [2]. Although the clinical and radiographic features of such neoplasms are usually distinctive, they can show some resemblance to those of CMFs. The most important histologic features of these entities are osteoid formation, which is coarse, lace-like bone present in periosteal osteosarcoma, and well-formed woven bone trabeculae surrounded by cellular fascicles of spindle cells in parosteal osteosarcoma, respectively.

Another benign lesion to differentiate is juxtacortical chondroma or synovial chondromatosis, especially when CMF involves the joint space, as in the current two cases. Juxtacortical chondroma consists of mature chondrocytes lying in lacunae with intervening chondroid matrix. Synovial chondromatosis exhibits nodules of hyaline cartilage, often surrounded by a rim of synovial lining. Juxtacortical chondroma and synovial chondromatosis lack a typical zonal pattern of CMF and show clustering of mature chondrocytes in hyaline cartilage matrix.

En-bloc resection is the mainstay of treatment in CMFs because curettage imposes about $25 \%$ risk of recurrence [1]. The prognosis after en-bloc resection is relatively good, though rare cases of recurrence and malignant transformation have been reported [2].

In summary, this study reported two rare presentations of juxtacortical CMFs in the small bones of the hands or feet. Our study indicates that juxtacortical CMFs, although rare, should be considered in the differential diagnosis of a surface-type osteolytic lesion that has well-circumscribed scalloped margins in the short tubular bones or an exophytic lesion radiologically misdiagnosed as soft tissue tumor in the interphalangeal joint.

\section{Ethics Statement}

This study was approved by the Institutional Review Board (IRB) of Kosin University Gospel Hospital with a waiver of informed consent (IRB No. 2021-09-030).

\section{Availability of Data and Material}

The datasets generated or analyzed during the study are available from the corresponding author on reasonable request.

\section{Code Availability}

Not applicable.

ORCID

Sun-Ju Oh https://orcid.org/0000-0001-6013-8579

So Hak Chung https://orcid.org/0000-0003-0689-0270

\section{Author Contributions}

Conceptualization: SJO, SHC. Data curation: SJO. Investigation: SJO, SHC. Supervision: SJO. Writing_original draft: SJO. Approval of final manuscript: all authors.

\section{Conflicts of Interest}

The authors declare that they have no potential conflicts of interest.

\section{Funding Statement}

No funding to declare.

\section{References}

1. Unni KK, Inwards CY. Dahlin's bone tumors. 6th ed. Philadelphia: Lippincott Williams and Wilkins, 2010; 50-9.

2. Baker AC, Rezeanu L, O'Laughlin S, Unni K, Klein MJ, Siegal GP. Juxtacortical chondromyxoid fibroma of bone: a unique variant: a case study of 20 patients. Am J Surg Pathol 2007; 31: 1662-8.

3. Jaffe HL, Lichtenstein L. Chondromyxoid fibroma of bone; a distinctive benign tumor likely to be mistaken especially for chondrosarcoma. Arch Pathol (Chic) 1948; 45: 541-51.

4. Takenaga RK, Frassica FJ, McCarthy EF. Subperiosteal chondromyxoid fibroma: a report of two cases. Iowa Orthop J 2007; 27: 104-7.

5. Estrada-Villasenor E, Cedillo ED, Martinez GR, Chavez RD. Periosteal chondromyxoid fibroma: a case study using imprint cytology. Diagn Cytopathol 2005; 33: 402-6.

6. Harrington KA, Hoda S, La Rocca Vieira R. Surface-type chondromyxoid fibroma in an elderly patient: a case report and literature review. Skeletal Radiol 2019; 48: 823-30.

7. Han JS, Shim E, Kim BH, Choi JW. An intracortical chondromyxoid fibroma in the diaphysis of the metatarsal. Skeletal Radiol 2017; 46: $1757-62$

8. Abdelwahab IF, Klein MJ. Surface chondromyxoid fibroma of the distal ulna: unusual tumor, site, and age. Skeletal Radiol 2014; 43: 243-6.

9. Slotcavage RL, Dickson BC, Ogilvie CM. Chondromyxoid fibroma involving the metacarpophalangeal joint. Orthopedics 2009; 32 orthosupersite.com/view.asp?rID=38065.

10. Wu CT, Inwards CY, O'Laughlin S, Rock MG, Beabout JW, Unni KK. Chondromyxoid fibroma of bone: a clinicopathologic review of 278 cases. Hum Pathol 1998; 29: 438-46.

11. Won KY, Lee J, Kim YW, Kim EJ, Kim SW, Park YK. Chondromyxoid fibroma of the ethmoid sinus complicated by a brain abscess: a case report and literature review. Korean J Pathol 2010; 44: 547-50. 